\title{
Dietrich Harth
}

\section{Grenzen der Wissenschaft}

Es gehört zu den Prinzipien der neuzeitlichen Wissenschaft, keine Grenzen anzuerkennen. Das Wissen dauernd zu erweitern, unablässig seine Anwendungsmöglichkeiten zu prüfen, aufhaltsame Krisen als schöpferische Pausen auf dem Weg ins Aufklärbare durchzustehen, äußerliche Hindernisse probeweise wegzudenken und nur jene Grenzen für rationale zu halten, mit denen sich spielen läßt - das sind die Fähigkeiten, die in den Verhaltenscodex des erfolgreichen Wissenschaftlers gehören. Doch die heute zutage tretenden Risiken haben dieser Norm die Unschuld genommen. Wissenschaftskritiker verlangen danach, die ungerichtete Expansion wissenschaftlicher Wissensproduktion einzudämmen und so zu begrenzen, daß die Folgen nicht einem ohnehin schon stark gefährdeten Leben weiter zur Last fallen.

Es ist die Frage, wie die geforderte, letztenendes politische Kontrolle $\mathrm{zu}$ institutionalisieren ist, welche Instanzen die Richtwerte bestimmen und ob überhaupt eine von außen kommende Begrenzung nicht die einst von der Sache her legitime und auch grundgesetzlich garantierte Freiheit wissenschaftlichen Handelns zerstören muß. Politische Fragen werden gern mit organisatorischen beantwortet. Doch, so ist zu fürchten, schließen solche 'Lösungen', da sie innerhalb des Systems verharren, einen grundlegenden Wandel aus. Dieser aber ist erforderlich, sollen die längst sichtbaren und die latent schwelenden $\mathrm{Ri}$ siken einer mithilfe der Wissenschaften technisch, physikalisch und chemisch umgebauten Umwelt nicht die Freiheiten zunichte machen, auf die sich der Forscherverstand so gern beruft. Wenn, was wohl absehbar ist, die Strategie der Schadensabwendung imperativisch in den Vordergrund tritt, so wird diese Freiheit vollends auf einen Reflex reduziert.

Mit dieser Vorbemerkung möchte ich andeuten, wie berechtigt mir die Angst und daraus abgeleitete moralische Empörung erscheinen, die sich - vielleicht noch nicht lautstark genug - angesichts bedenkenlos durchgesetzter wissenschaftlich-technischer Projekte äußern. Sieben- 
tausend amerikanische Wissenschaftler, so war kürzlich zu lesen, haben sich geweigert, an der "Strategic Defense Initiative" des Weißen Hauses mitzuarbeiten. Wobei zu bedenken ist, daß in den USA derartige Staatsaufträge in der Regel nicht als Eingriff in die Freiheit der Wissenschaften interpretiert werden. Unterstellen wir einmal als Ablehnungsmotiv die Angst vor einer neuen und neuartigen Rüstungseskalation, so könnte diese Weigerung als Anzeichen für ein Raisonnement gewertet werden, das mit common sense auf den Systemzwang reagiert. Ein Heraustreten aus dem System via reflexionis oder gar via rebellionis ist das noch nicht. Dafür gibt es die Philosophen und die Bewegungen der Gegenkultur.

Es ist aber ein Unterschied, dem common sense zu folgen oder nach den Möglichkeiten einer Korrektur zu fragen, die dem Wissenschaftsdiskurs selbst innewohnen. Ist denn dieser Diskurs so blind gegenüber den praktischen Folgerungen, die aus ihm abgeleitet werden? Ist die wissenschaftlich abgezirkelte Erkenntnisrationalität tatsächlich so sehr versteinert gegenüber den praktischen Bedürfnissen, von denen sie einst ausging? Das sind Fragen, die auf Grundlage und Legitimität des wissenschaftlichen Denkens zielen, und daher philosophische Antworten beanspruchen. Karl Jaspers hat den Vorteil, daß er nicht nur als Philosoph über die Wissenschaften sprach, sondern selbst als praktizierender Arzt an prominenter Stelle in der klinischen Forschung tätig war und ein bedeutendes Fachbuch zur Sache geschrieben hat. Umso mehr weckt die schon früh von ihm zur Sprache gebrachte Wissenschaftskritik den Wunsch nach Kenntnis der von ihm vorgetragenen Lösungsvorschläge zu den genannten Problemen.

Zunächst versuche ich einen Begriff von dem zu geben, was Jaspers unter "Wissenschaft" verstand. In einem weiteren Schritt skizziere ich den von ihm eingeschlagenen Weg an den, ja über die Grenzen wissenschaftlichen Denkens hinaus. Dann erst diskutiere ich die Relevanz seiner philosophischen Antworten für die aktuellen Probleme.

Jede Analyse des Wissenschaftsbegriffs muß von dem ausgehen, was "Wissen" bedeuten kann. Schon im Rahmen der klinischen Psychiatrie hat sich Jaspers die Frage vorgelegt, was in der Diagnose seelischer Krankheiten Gegenstand des Wissens, was Gegenstand der Vermutung sei. Ein Problem, dessen methodologische Entfaltung in der Allgemeinen Psychopathologie (1913) nachzulesen ist. In diesem 
Buch folgt Jaspers - darauf hat Dieter Henrich hingewiesen (1986, 207ff.) - Grundsätzen, die er von Kant und Max Weber übernommen hat. Webers Lehre der Idealtypen diente ihm zum Entwurf einer Methodik, die niemals verkennt, daß das von ihr angestrebte Wissen abstrahierenden Verkürzungen und Fiktionen zu verdanken ist. Kants Ideenlehre legte ihm nahe, das Leben als ganzes, also auch die Person des seelisch Erkrankten, wie eine Idee zu betrachten, die einer vollständigen wissenschaftlichen Erklärung sich widersetzt. Gleichwohl treibt diese Idee dazu an, die Bemühungen um das Verständnis der Lebenstotalität niemals aufzugeben, sondern dauernd noch zu verstärken.

Die beiden nach dieser Theorie innerhalb des Wissenschaftsdiskurses zu unterscheidenden Verfahren des Erklärens und des Verstehens entsprechen in etwa der in der philosophischen Tradition geläufigen Trennung zwischen technischem Wissen einerseits und Allgemeinwissen andererseits. An diese Tradition knüpfen auch die ersten Sätze von Jaspers' Psychologie der Weltanschauungen aus dem Jahre 1919 an. "Was ist Weltanschauung?" fragt er dort und antwortet:

Etwas Ganzes und etwas Universales. Wenn z.B. vom Wissen die Rede ist: nicht einzelnes Fachwissen, sondern das Wissen als eine Ganzheit, als Kosmos (PW, 1).

Es läßt sich darüber streiten, ob der Begriff des Wissens mit dem der Weltanschauung überhaupt kommensurabel ist. Unsicherheit darüber hat wohl auch Jaspers empfunden, da er fortfährt:

Weltanschauung ist nicht bloß ein Wissen, sondern sie offenbart sich in Wertungen, Lebensgestaltung, Schicksal, in der erlebten Rangordnung der Werte (ebd.).

Es sind also, mit Max Weber zu reden, die "Wertideen", die in den Weltanschauungen zu Buche schlagen, und damit sind wir bei einer Form der Erfahrung, die gerade nicht in der Rationalität methodisch disziplinierter Erkenntnis aufgeht. Diese Erfahrung steht nämlich in einem besonderen Spannungsverhältnis zum Wissen und wird von Jaspers mit Begriffen umschrieben, die an die theoretische Einstellung des antiken Philosophen gemahnt: "Ein Leben in der Schwebe, im labilen Gleichgewicht, schauend, staunend..." (PW, 332). In der Moderne ist dieser theoretischen Haltung jedoch verwehrt, im Gedanken an je- 
nen unveränderlichen Kosmos zur Ruhe zu kommen, der einst die Welt im Innersten zusammenhielt. Rufen wir uns die klassischen Begriffe in Erinnerung, um den Unterschied deutlich werden zu lassen!

In der antiken Philosophie - ein bekanntes Beispiel ist Platons Timaios - bedeutete das Wort "theoria" das Anschauen nicht der vergänglichen Dinge, sondern der unvergänglichen kosmischen Ordnung. Wer sich theoretisch verhielt, der widmete sein Denken dem ewigen Sein und konnte darauf bauen, daß seine Seele sich den unwandelbaren Ordnungsstrukturen, die er anschaute, assimilierte. Theorie war jedoch nicht vom Leben geschieden. Sie war vielmehr dessen höchste Form und mit der religiösen Erfahrung verwandt. Die Erkenntnis, die der theoretischen Schau entsprang, bestand nicht in fachlichem oder technischem Wissen, sie entsprach überhaupt keiner Fertigkeit. Vielmehr brachte sie jenes allgemeine Wissen des Ganzen hervor, das, um bildend wirksam zu werden, gelebt werden muß. Daher auch die enge Bindung der Theorie an eine Lebensform, an den Bios theoretikos.

Zwar kann Jaspers, spricht er vom "Leben des Geistes", an diese Tradition anknüpfen, indem er unbeirrt den hohen Rang der geistigen Lebensform behauptet. Doch besitzt der Logos kein Heimatrecht mehr in einem unvergänglichen Kosmos. Das "Ganze" der Weltordnung ist seit der kritischen Destruierung metaphysischer Weltbilder kein Gegenstand für Erkenntnis und damit entfällt der Grund für die transzendente Gewißheit der philosophischen Vordenker. Daher befindet sich das Leben des Geistes allenfalls in einem schwankenden, Veränderungen geradezu heraufbeschwörenden Zustand. Denn anders gesagt: Es ist identisch mit der unablässigen Suche nach einer "umgreifenden" Ordnung, die den unsteten Ordnungen der gelebten Erfahrung einen Sinn zu geben vermag. Das einzige, was dieser von Angst, Verzweiflung und Tod bedrohten Suche Halt verleiht, ist der Glaube, daß es dieses andere - diese das Partikulare übergreifende Ordnung geben muß. Glaube aber steht - nach überlieferter Denkgewohnheit nicht nur im Gegensatz zum Wissen, weshalb das geistige Leben nicht auf ein Erkennen dessen, zielt, was ist. Glaube ist vielmehr - so schreibt Jaspers selbst - "ein Akt, der überhaupt erst (auch) die Bewe- 
gung zum Wissen hin möglich und sinnvoll macht" (PW, 337). Zugleich aber soll der Glaube auch Geist" sein, wie es an derselben Stelle in lapidarer Verunklärung heißt."

Als transzendentale Bedingung des Wissenwollens hat dieser Glaube (alias Geist) nicht unmittelbar mit jenem gegenständlichen Wissen zu tun, das die Wissenschaften als handfestes Ergebnis hervorbringen. Nur dort, wo diese über die selbstgezimmerten Gehäuse ihrer Fachkenntnisse und spezialisierten Aussagen hinausschauen, wo sie z.B. genötigt sind, über den Sinn ihrer rationalen Wirklichkeitsproduktion nachzudenken, dort stoßen sie - nach Meinung des frühen Jaspers - notwendigerweise auf die im Glauben bezeugte Bewegung des Geistes. Gewißheit und Ungewißheit, Wissen und Glaube stehen nach dieser Idee nicht nur in einem Gegensatz, sie bedingen einander vielmehr. Denn der Glaube bezeichnet die Grenze, die das Wissen vom Nicht-Wissen trennt.

Bleibt die damit aufgeworfene Frage nach dem Verhältnis von Philosophie und Wissenschaft in Jaspers' Frühwerk noch peripher, so wird sie im Hauptwerk von 1932, in der Philosophie, zu einem zentralen Thema.

Die Form von Jaspers' Philosophie - und darauf hat jede Deutung Rücksicht zu nehmen - ist ihrem Gehalt nicht äußerlich. Sie unterscheidet sich grundlegend von der Form des wissenschaftlichen Diskurses, beansprucht jedoch eine eigene Systematik. Schon der Psychologie der Weltanschauungen lag eine Komposition zugrunde, die in einer Bewegung, die von Kapitel zu Kapitel sich wiederholte, vom Sinnlich-Gegenständlichen zum Gegenstandslosen und Spekulativen führte. Diese Form bestimmt, mit größerer Strenge durchgeführt, auch den Aufbau der Philosophie. Sie deckt sich mit jener von Kant eingeführten "Methode" des Philosophierens, der Jaspers die Schlüsselmetaphern des "Überschreitens" und "Transzendierens" verdankt. Doch interessiert ihn nicht das, was die transzendentale Methode in den Erkenntnisbesitz des Denkenden überführt, sondern allein die Bewegung, der Vollzug des Überschreitens. Wie der Tanz nur im Getanztwerden zur Darstellung kommt, so ist genuines Philosophieren eins mit der Bewegung des Transzendierens. Es gleitet, da es nichts Trennendes anerkennt, gleichsam auf der Grenze entlang, die zwischen allen Gegensätzen verläuft, also auch zwischen den Begriffen einer wirklichen 
und einer möglichen Welt, die Jaspers terminologisch als "Dasein" vom "Sein" unterscheidet. Im Vollzug des Überschreitens werden solche Unterscheidungen nichtig: "Transzendieren vom Dasein zum Sein ist weder als In-der-Welt-sein noch als Außer-der-Welt-sein" (P,37). Eben das resultierende Dazwischen-Sein aber verleiht, wird es ins Bewußtsein gehoben, der wirklichen Welt etwas Scheinhaftes. Positiv gewendet: Das Dasein wird nun als ein unendlich auslegbarer Pseudotext, als - wie Jaspers sagt - "Chiffreschrift", wahrgenommen. Allerdings gibt es für die Entzifferung keinen passenden Schlüssel, so daß die Auslegungsarbeit niemals am Ziel eines bestimmten Sinnes ankommen kann.

Wer sich auf diese Weise auslegend zur Welt verhält, der hat nach Jaspers einen "Akt der Freiheit" - früher hieß es noch: "Akt des Glaubens" - vollzogen $(P, 37)$. Auch zu seinem persönlichen Dasein verhält er sich anschauend und auslegend und vermag mithilfe des Transzendierens in den unbestimmten Bildern einer potentiell anderen Existenz zu lesen. Der Vollzug des Überschreitens schlägt in Erfahrung um, und diese Erfahrung soll spürbar machen, wie zufällig das ist, was das gewöhnliche Bewußtsein für das Wirkliche hält.

Es ist keine Frage: Was diese Art des Philosophierens intendiert, das ist mit den Absichten wissenschaftlicher Erkenntnis nicht vereinbar, ja es scheint diesen direkt entgegengesetzt zu sein. Das gilt nicht nur für den sogenannten Satz vom Widerspruch, sondern für alles, was das moderne Gesicht der Wissenschaften geprägt hat: Professionalisierung des Forschungsprozesses, Spezialisierung des Wissens, Zweckrationalität der Verfahren, technische Anwendbarkeit usf. Die damit verbundenen Leistungen erkennt der Philosoph durchaus an, sind sie doch unübersehbar. Sie haben nicht nur die Lebenswelt verändert sondern auch die Voraussetzungen jenes Philosophendiskurses, der von überlieferten Denkgewohnheiten zehrt. So hat Wissenschaft als Kritik dem Vertrauen in die absolute Welterkenntnis und in ganzheitliche Weltbilder den Boden entzogen, und als Produktivkräfte greifen bestimmte, technisch konvertible Wissenschaftserkenntnisse selbst in unsere privatesten Erfahrungen ein.

Aber aus der unabschließbaren Bewegung dieser Prozesse zieht die philosophische Erkenntnis den Schluß, daß selbst dem methodisch zweckhaften Denken und Tun Grenzen gezogen sind, die dieses nicht 
aus eigener Kraft überschreiten kann. Darin steckt auch ein Hinweis auf die Gefährdungen, die von den Wissenschaften ausgehen. Denn ihre Erfolge verführen zu jenem neuen Aberglauben, der an die Stelle der alten metaphysischen Gewalten die scheinbar unumstößliche Gewißheit wissenschaftlicher Tatsachenfeststellungen setzt. Diese Gefahr abzuwenden, ist ein durchgehendes Thema von Jaspers' zeitkritischen Schriften Die geistige Situation der Zeit (1931), Vom Ursprung und Ziel der Geschichte (1949) und Die Atombombe und die Zukunft des Menschen (1958).

Um dem beschworenen Aberglauben und seinen schlimmen Folgen für die Verfassung der Lebenswelt zu entgehen, bedarf es, davon ist Jaspers überzeugt, umso notwendiger des Philosophierens in dem angedeuteten gnostischen Sinn. Philosophie als Wissenschaft verharrt innerhalb der Grenzen der institutionalisierten Diskurse. Die Existenzphilosophie will mehr. Ihr Anspruch ist nicht außerwissenschaftlicher, sondern überwissenschaftlicher Natur. Im Überschreiten des wissenschaftlichen Denkens erfüllt sich ihre Aufgabe, nicht in dessen Verdrängung oder in einem sich immer höher aufstufenden Wissen.

Statt noch einmal zu tun, was Wissenschaft getan hat [so ist in der Philosophie zu lesen], wird unter Voraussetzung ihres Wissens mögliche Existenz evoziert ( $P, 665)$.

Dieser Satz enthält einen Hinweis auf die Grenze, die zwischen Wissenschaft und Philosophieren verläuft. Wir müssen uns, um diese Grenze mit Jaspers denken zu können, noch einmal dem zuwenden, was wissenschaftliches Wissen im Rahmen der Existenzphilosophie bedeutet. Auch Wissenschaft soll ihrer Idee nach Lebensform sein. Das ist wichtig, weil darin die Kritik an den modernen Professionalisierungserscheinungen gründet. In den Denkschriften zur Idee der Universität von 1923 und 1946 ist ausgeführt, wie diese Lebensform sich zu dem, was nach Idee und institutioneller Wirklichkeit möglich sein soll, verhält. Auf dieses Verhältnis zur Institution möchte ich hier jedoch nicht eingehen, da mein Interesse dem Begründungszusammenhang gilt.

Spricht Jaspers von "Lebensformen", so spielt er auf die Bedürfnisse an, die allen menschlichen Institutionen zugrunde liegen. Er führt zum Beispiel die mögliche Lebensform wissenschaftlicher Praxis auf 
einen "Antrieb" des Immer-mehr-wissen-wollens zurück, den er - ein Wort Nietzsches abwandelnd - den "Willen zum Wissen" nennt. Dieser Wille zum Wissen dient der Orientierung in der Welt. Er ist der Motor einer zielstrebigen Erkenntnisunruhe, die jedoch niemals bei einem 'absoluten' Wissen ankommen kann. In den Einzelwissenschaften hat dieses Wissen-Wollen seine systematische Fassung erhalten. Hier ist es auf Allgemeingültigkeit und zwingende Gewißheit aus, Ideale, die normierend auf die Bestimmung der Gegenstände und Untersuchungsmethoden zurückwirken. Allgemeingültigkeit fordert die selbstkritische Einklammerung der Subjektivität, zwingende Gewißheit: d.h. Strenge der Kategorien, Reinheit der Methoden und theoretische Konstruktion des Objekts. Gerade diese Zurichtungen aber sind es, die Jaspers als Zugeständnisse an die Überwindbarkeit der Erkenntnisgrenzen durchschaut. Gewiß, faktisch orientieren die Wissenschaften wie keine andere Lebensform das Dasein in der Welt. Doch ihr Konstruktivismus verweist auf einen Lebenssinn, der die Wirklichkeit anders erfährt, nämlich auf die gelebte Wirklichkeit. Die "unmittelbare" oder gelebte "Wirklichkeit" ist, so heißt es in der Philosophie, mehr als das "reine Objekt" jener "empirischen Wirklichkeit", mit der sich die Wissenschaften befassen (P, 79). Diese konkretisieren stets nur einzelne Wirklichkeiten, niemals die Wirklichkeit selbst, oder, wie Jaspers schreibt, "das Ganze der Welt" (ebd.). Zwar streben die Einzelwissenschaften über den Pluralismus ihrer Erkenntnisse hinaus, sie zeigen auch in ihren formalen Strukturen die Tendenz zur Einheit des Wissens. Doch ist die Unabschließbarkeit ihrer Erkenntnisse ein sicheres Zeichen dafür, daß es nicht in sich selbst gründet, sondern relativ und perspektivisch gebrochen ist.

So können die Wissenschaften - trotz ihrer Fortschritte in der Aufklärung partikularer Wirklichkeiten - nicht über die Grenzen hinaus, die das Fachwissen ihnen steckt. Es läßt sich sogar genauer bestimmen, welcher Art diese Grenzen sind. Auf der einen Seite ist es die prinzipielle Unbegreifbarkeit der bewegten Materie, auf der anderen Seite die personal begründete, völliger Objektivierung entzogene Freiheit des Individuums. Mit Jaspers' eigenen Worten:

Die Naturwissenschaften suchen das Undurchdringliche in Gesetzlichkeit und Theorie einzufangen, die Geisteswissenschaften konstruieren die Werke und Erscheinungen der Freiheit zu be- 
wußter Vergegenwärtigung in ihrer Gesetzlichkeit und normativen Bedeutung. Die absolute Grenze aber ist für die Naturwissenschaft das dunkle schlechthin Andere, für die Geisteswissenschaften die Freiheit der Existenz als Ursprung der Kommunikation $(\mathrm{P}, 126)$.

Über den Sinn, das Warum und Wozu der Wissenschaften etwas zu sagen, macht das Überschreiten dieser Grenzen notwendig und kann nur geschehen, indem der Wissende - wie Sokrates - eingesteht, daß er nicht weiß. Das Denken soll ausdrücklich die Welt der sicheren Begriffe und allgemeingültigen Aussagen hinter sich lassen, um Einsicht in die Relativität und perspektivische Gebundenheit aller wissenschaftlichen Erkenntnisse zu gewinnen. Mögen die Experten auch Selbstkritik üben, und nach Jaspers ist diese ein integrales Element der fachgebundenen Erkenntnis, so führt sie diese doch nicht an ihre Grenzen, da Selbstkritik als rationale Diskursform noch im Dienst am Wissen befangen bleibt.

Das methodologische Bewußtsein [bemerkt Jaspers] entfremdet alles Denken und Handeln sich selber (GO, 438).

Eine Möglichkeit, diese Befangenheit abzustreifen, liegt in der Ironie, zumal in ihrer sokratischen Spielart. Diese Ironie besitzt zwar einen "Sinn für Wirklichkeit", bringt aber alles, was nach objektiver Fixierung im Wissen des Wirklichen strebt, in die Schwebe (P,539). Ironie klagt, so ist das zu verstehen, zugleich mit der Wirklichkeitsbehauptung die Mehrdeutigkeit des Behaupteten ein. Sie liegt also schon auf dem Weg zu jenem überschreitenden Denken, das Jaspers als den Königsweg in die Negativität der Freiheit - will sagen: in die Verneinung der Macht des Faktischen - umschrieben hat. Wer diesen Weg geht, der macht sich frei von der Partikularität des Wissens, er überschreitet die Grenze des gegenständlichen Denkens. Bezeichnenderweise soll die damit identische Freiheit auf Begriffe verzichten können. Ihr Medium ist zwar, wie Jaspers betont, auch "Sprache", doch es ist die Sprache der vieldeutigen "Chiffren", deren Bedeutung - auch wenn diese dauernd gesucht wird - nicht aussagbar ist.

Der systematische Aufbau von Jaspers' Philosophie spiegelt diesen Weg des Denkens von der Orientierung in der Wirklichkeit über das Erhellen möglicher Existenz bis zum "Lesen" der Symbole und Chif- 
fren wider. Ich habe den Eindruck, daß hier der antike Weg des Denkens von den Dingen bis hin zur Schau der Ideen - Ideen als die wahren Urbilder der scheinhaften, gegenständlichen Welt verstanden noch einmal nachvollzogen werden soll. Dieser Weg ist freilich in der Moderne verschlungen und hindernisreich, da er über Wissenschaften führt, die sich von der Philosophie losgesagt haben. Aber seine Topographie bewahrt noch die Spuren jener existenzverwandelnden kontemplativen Haltung, die einst zum Inhalt des bios theoretikos gehörte. Die wirklichkeitsschaffende Macht wissenschaftlicher Rationalität vermag diese Philosophie jedoch nicht zu brechen. Sie verweist sie allenfalls in die Schranken eines zweckhaften Tuns, das längst den metaphysischen Schein der Dingwelt entzaubert hat.

Hier liegt nun auch eine Schwierigkeit der Existenzphilosophie in ihrem Verhältnis zum wissenschaftlichen Denken. Sie erscheint, um es formelhaft auszudrücken, als das Negativ der wissenschaftlichen Zweckrationalität. Sie greift diese nicht mit den Mitteln der Analyse an und übt insofern auch keine Kritik in des Worts strenger Bedeutung. Ihr Habitus ist im Grund polemisch und ihre Ausgangsbasis eine Ontologie der Verzweiflung, die das Leben und seine konkreten Ordnungen unter der Bedingung der Endlichkeit denkt. Ihre Mitteilungsformen sind darüber hinaus nicht sachgebunden. Sie lehrt nichts, gibt keine Richtung an, spendet keinen Trost und versagt sich jedem billigen Optimismus. Stattdessen appelliert sie - das ist alles. Sie will Haltungen, nicht Begriffe ändern - das ist viel. Jaspers hat persönlich niemals die Vorbildlichkeit des Weisen und Lehrers verleugnet.

Der "moderne Mensch", notierte er später einmal und bezog sich dabei auf Max Weber, habe "wirklich ernst gemacht mit der grenzenlosen [!] Redlichkeit", er setze sich dem Kampf der Mächte aus, lebe leidenschaftlich und kämpfe mit sich selbst ohne Ziel (Arendt/Jaspers 1985, 671). In dieser Notiz spielt der Philosoph nicht nur auf die Endlichkeit des menschlichen Lebens an, die als finis ultima des Willens zum Wissen zu betrachten ist. Er faßt hier auch in exemplarischer Verkürzung jenes Ethos zusammen, das der Wissenschaftler ausbilden muß, will er die Grenzen seines eigenen Tuns denken. Nicht die Praxis ist zuerst zu verändern, sondern die Haltung. Das Bild des Kämpfers, der sich nie am Ziel weiß, der also das Scheitern annimmt, verleiht der 
geforderten Haltung heroische Züge. Wo die wissenschaftliche Rationalität die Freiheit der Existenz bedroht, etwa im Bündnis mit dem "Verhängnis" moderner Technik, dort - so argumentiert Jaspers - kann Abhilfe nicht aus dem kommen, was die Bedrohung verursacht hat: aus der zweckgebundenen Rationalität. Nur der "Sprung" in die Existenz führe zur Umkehr. Es ist also eine andere als die wissenschaftliche Vernunft erforderlich, sollen die negativen Folgen des Bündnisses zwischen Wissenschaft und Technik vermieden werden. Auch terminologisch ziehen Jaspers' spätere Schriften eine Trennungslinie zwischen beiden Formen der Rationalität, indem sie die Wissenschaft dem "Verstand", das philosophische Transzendieren aber der "Vernunft" zuordnen (AZM, 293).

Als Bewegung, nämlich als Überschreiten, entzieht sich diese Vernunft der genauen Bestimmung. Andererseits soll sie zur "Umkehr" anhalten, soll also praktisch wirksam werden. "Praktische Einsicht und Transzendieren vollziehen sich im Raum der Vernunft", heißt es dazu in Die Atombombe und die Zukunft des Menschen; und weiter:

Von der Umkehr aus der gewohnten, sich in sich genügsamen Denkweise des Verstandes in die der Vernunft hängt ab, was aus dem Menschen wird (AZM, 298).

Unter anderen Begriffen zeichnen diese Sätze noch einmal nach, was die frühere Philosophie bereits zum Thema hatte: das Überschreiten des weltorientierenden Denkens in einem Akt der Freiheit. Nur daß nun, angesichts des möglichen Selbstmords der Gattung, die Frage nach den unmittelbar praktischen Wirkungsmöglichkeiten unabweisbar geworden ist.

Soll die Welt nicht den Gesetzen der wissenschaftlich-technischen Rationalität überlassen bleiben, so muß sich der von der 'anderen' Vernunft intendierte Freiheitsakt auch in eine andere Lebensordnung umsetzen lassen. An dieser Stelle kommen nun jene Tugenden einer kämpferischen Haltung ins Spiel, die Jaspers unter dem Titel "Ethos wahrer Polemik" zusammengefaßt hat. Programmatisch ist der folgende Satz:

Moral, Recht, Opfermut sind selber Vernunft, weil Vernunft nur in ihnen, nicht als bloße Vernunft wirklich werden kann (AZM, 254). 
Resümieren wir noch einmal die wichtigsten Züge dieser 'praktischen' Philosophie: Der Wille zum Wissen bringt kein Ethos hervor, das imstande wäre, die Gefährdungen, die von der technischen Nutzanwendung des rational Erkannten ausgehen können, aufzuhalten oder gar umzukehren. Wo dieser Wille die Faktizität einer Organisation angenommen hat, in den wissenschaftlichen Institutionen, dort herrscht "neutrale Gleichgültigkeit" gegenüber den ethischen Fragen nach einer menschenwürdigen Existenz. Wissenschaft "als solche", so lautet Jaspers' Vorwurf, ist "weder menschlich noch vernünftig" (AZM, 279). Mit einem Wort: Der Philosoph spricht dem wissenschaftlichen Diskurs die Fähigkeit ab, von innen heraus, etwa auf dem Weg der kritischen Selbstreflexion, jenes Vernunftinteresse zurückzugewinnen, das eine mißverstandene Reinheitsnorm verschüttet hat. Die Antwort auf unsere eingangs gestellte Frage ist demnach eindeutig: Es gibt keine Korrekturmöglichkeiten innerhalb der Wissenschaften! Nur in den seltenen Fällen, in denen der Diskurs vom Ethos einer freundschaftlichen Forscherbeziehung durchdrungen ist, soll er die Sphäre der Gleich-Gültigkeit verlassen.

Zwar schließt das nicht aus, daß der Wissenschaftler sein Metier 'überschreitet', indem er den "Sprung" ins Philosophieren wagt, aber es drängt sich doch vor allem die Frage auf, warum Jaspers Aufklärung und Kritik, die bekanntlich beide zu den Geburtshelfern der modernen Wissenschaften gehören, mit solcher Geringschätzung behandelt hat. Gehen beide Formen der Weltorientierung denn völlig in einem ethisch neutralen Diskurs des Nutzenkalküls auf? Anders gefragt: Ist das zweckbezogene und vergegenständlichende Denken wirklich der einzige Typus wissenschaftlicher Rationalität? Und noch etwas: Ist nicht in Jaspers' Begriff der "Perspektivierung" schon der Hinweis enthalten, daß selbst die reinste wissenschaftliche Erkenntnis an einen Standpunkt innerhalb lebensweltlicher Interessen gebunden ist, daß um es mit anderen Worten zu sagen - subjektive sowie soziale Erfahrungen an der Wahl der Gegenstände und am Entwurf der Forschungsfragen konstitutiv beteiligt sind?

Diese Fragen stehen hier, zumal sie auch mehr oder weniger rhetorisch gedacht sind, nicht zur Beantwortung. Ich möchte nun vielmehr den letzten angekündigten Schritt tun, um auf Jaspers' eigene Ant- 
worten zu sprechen zu kommen, die er Ende der fünfziger Jahre im Rahmen einer Kontroverse vorgetragen hat, die auch heute noch nicht zum Abschluß gekommen ist.

Zunächst einige Anmerkungen zur Situation: Am 12. April 1957 veröffentlichte eine Gruppe von achtzehn Physikern, unter ihnen Max Born, Otto Hahn, Werner Heisenberg, C.F. von Weizsäcker, in Göttingen eine Erklärung, die mit dem Satz beginnt:

Die Pläne einer atomaren Bewaffnung der Bundeswehr erfüllen die unterzeichneten Atomforscher mit tiefer Sorge.

Damals waren solche Pläne von Adenauer und seinem Verteidigungsminister F.J.Strauß auf den Tisch gelegt worden. Vor allem Adenauer hatte durch den verharmlosenden Vergleich der taktischen Atomwaffen mit der konventionellen Artillerie zur Beunruhigung der Wissenschaftler beigetragen. Deren öffentliche Erklärung wurde als eine ganz ungewöhnliche, wenn nicht unerhörte Einmischung in die Politik empfunden, und der Kanzler konterte, indem er ihnen die politische Urteilsfähigkeit aberkannte. Damit bestätigte er eine Erwartung, die von den Wissenschaftlern schon im voraus formuliert worden war. Warum sie dennoch das Wort ergriffen, das begründeten sie auf folgende Weise:

Unsere Tätigkeit, die der reinen Wissenschaft und ihrer Anwendung gilt [...], belädt uns aber mit der Verantwortung für die möglichen Folgen dieser Tätigkeit. Deshalb können wir nicht zu allen politischen Fragen schweigen (Weizsäcker 1957, 51).

Das notorische Schweigen der Wissenschaft in politischen Fragen zu brechen, war aber nicht das einzige Ziel des Göttinger Manifests. Es hatte auch etwas zur Kooperationsbereitschaft der Physiker zu sagen. Keiner der Unterzeichner wäre bereit, so hieß es im Text,

sich an der Herstellung, der Erprobung oder dem Einsatz von Atomwaffen in irgendeiner Weise zu beteiligen. Gleichzeitig betonen wir [so lautet der letzte Satz], daß es äußerst wichtig ist, die friedliche Verwendung der Atomenergie mit allen Mitteln zu fördern, und wir wollen an dieser Aufgabe wie bisher mitwirken (Weizsäcker 1957, 52).

Den Wissenschaftlern war klar, daß beim damaligen Stand des Wissens auch ohne ihr Mitwirken Atomwaffen entwickelt und herge- 
stellt werden konnten. Daher trat umso schärfer die politische und moralische Bedeutung ihrer Erklärung hervor. Carl Friedrich von Weizsäcker, der Philosoph in der Runde der Unterzeichner, erläuterte die Grenze des bloß politischen Kalküls an dem Satz, daß Waffen, deren Anwendung Freund wie Feind fürchten muß, nur dann stabilisierend - im Sinne eines "Gleichgewichts des Schreckens" - wirken können, wenn ihr Einsatz auch ernsthaft geplant wird. Dies mußte und muß damals wie heute die Hoffnungen auf eine rein politische Lösung der Konflikte beeinträchtigen, da die Blöcke in Ost wie West nicht die geringste Bereitschaft zeigten, das selbstzerstörerische Spiel um die Macht mit anderen als militärischen Mitteln zu beenden. Was das Göttinger Manifest in dieser Situation zu sagen hatte, das sollte daher, so Weizsäckers Kommentar, als Appell an eine neue Planungsethik verstanden werden. Diese stand unter dem Motto: Zuerst kommt der Mensch, dann kommt die Planung. Mit Weizsäckers Worten:

Die Reifezeit der Technik - wenn es zu einer solchen kommen wird - wird ihre Reife in der Distanz zum Apparat, in der Fähigkeit zum ruhigen, überlegenen Verzicht auf gewisse technische Möglichkeiten beweisen müssen, kurz, in der Unterordnung des Plans unter den Menschen (Weizsäcker 1957, 10).

Gerade das aber, wie auch der Gehalt der von Weizsäcker verkündeten Ethik forderte den Widerspruch von Jaspers heraus, den er in seinem 1958 veröffentlichten Buch Die Atombombe und die Zukunft des Menschen mit einiger Ausführlichkeit dargelegt hat. Auch die Physiker hatten eine Grenze bezeichnet, nämlich die Grenze zwischen der Sachrationalität des Politikers und der Verantwortung des Wissenschaftlers vor dem, was sein Sachverstand an Möglichkeiten zur technischen Umformung, wenn nicht Vernichtung des Lebens hervorgebracht hat. Sie hatten also nicht - was Jaspers kritisch vermerkte - die Grenzen des wissenschaftlichen Sachverstandes thematisiert, sondern nur diejenigen, die aus Gründen der Selbsterhaltung dem instrumentellen Handeln in Politik und Technik zu ziehen sind. Sie hatten darüber vergessen, daß sie selbst in ihrem Beruf, als dessen Repräsentanten sie in der Öffentlichkeit aufgetreten waren, eben jenes zweckhafte Denken ausbilden und kultivieren, dessen Kurzsichtigkeit ihre Erklärung verurteilte. 
Jaspers lag es fern, die guten Absichten der achtzehn Physiker anzuzweifeln. Doch er sah den Erfolg der Erklärung weder im Entschluß, die Mitarbeit aufzukündigen noch in ciem von Weizsäcker nachgelieferten Aufruf, das technische Handeln nach Maßgabe ethischer Grundsätze zu verändern. Der Erfolg lag für ihn nicht im Inhalt, sondern in den Reaktionen, die das Manifest in der Öffentlichkeit, besonders bei Politikern hervorgerufen hatte. Was die Physiker forderten, die Bundesrepublik von Atomwaffen frei zu halten, das wollte Jaspers nicht gelten lassen, da die Erfüllung auf eine Schwächung des westlichen Bündnisses hinauslaufe. Die Physiker haben, so lautet sein Vorwurf, ohne Rücksicht auf die realpolitische Situation nach grundlegender Änderung der Politik verlangt. Neutralität aber kam für Jaspers überhaupt nicht in Frage. Die Garantie für die, wie er sich ausdrückte, "Selbsterhaltung des freien Abendlandes" (AZM, 273) lag für ihn wie für Adenauer (den der Philosoph im übrigen nicht selten kritisierte) in einer starken militärischen Verteidigung. Und dazu gehörte, wie er mit großer Selbstverständlichkeit annahm, die Stationierung taktischer Atomwaffen in den Staaten des Nato-Bündnisses.

Was Jaspers verschwieg, das sind die pragmatischen, politisch durchaus ernst zu nehmenden Vorschläge von Weizsäckers, die auf einen Atomtest-Stop und auf Rüstungskontrollen hinausliefen, Vorschläge, deren Aktualität bis heute ungebrochen ist. Es ist kaum verständlich, warum Jaspers sie nicht erwähnt hat. Der Verdacht liegt nahe, daß er absichtlich in der Schwebe lassen wollte, ob die westliche Rüstung dem Angriff oder der Verteidigung diente. Immerhin, es erschien ihm unproblematisch, dort die Anwendung von Gegen-Gewalt zu empfehlen, wo ein Glaubensinhalt mit Gewalt durchgesetzt werden sollte. Wie nah er in dieser Frage der Ideologie des Kalten Krieges stand, das belegen folgende Sätze:

Der Wissenschaftsaberglaube des Marxismus tritt absichtlich und faktisch mit Gewalt auf. Der Gewalt muß, wer sich behaupten will, Gewalt entgegensetzen, der gesteigerten eine ebenso gesteigerte Gewalt. Nichts in der Welt, auch nicht und am wenigsten der philosophische Glaube der Vernunft, kann sich im Dasein halten ohne Opfermut (AZM, 369).

Es ist bemerkenswert, daß Jaspers den Ost-West-Konflikt nicht in erster Linie als Auseinandersetzung um Machtpositionen interpretierte, 
sondern als einen Glaubenskrieg, in dem die Seite des Gegners jene wissenschaftliche Rationalität verabsolutierte, die im eigenen Lager Gegenstand der philosophischen Kritik war. Nur diese Perspektive rechtfertigte es, von einem übergeordneten moralischen Standpunkt aus in die tagespolitischen Kontroversen einzugreifen. Der Kalte Krieg der Ideologien, nicht aber die Politik lockte den Philosophen aus der Reserve. Indessen liegt in den zitierten Äußerungen etwas Unklares und Beunruhigendes, das einer grundsätzlichen Prüfung bedarf. Diese ist hier nur in groben Umrissen zu leisten und verlangt, daß wir von der politischen wieder zur philosophischen Fragestellung zurückkehren.

Das Beunruhigende liegt in der Verbindung zwischen Vernunft und Opfermut alias Gewalt. Denn Vernunft bezeichnet an dieser Stelle nicht die Bewegung einer niemals zum Stillstand gelangenden Verständigung über den Sinn der Existenz. Sie soll auch, so verlangt es der Aufruf zur Gegen-Gewalt, in bestimmten Situationen von ihrem Gegenteil, vom instrumentellen Handeln, Gebrauch machen dürfen. Darin liegt freilich eine starke Paradoxie. Wenn Vernunft um der Selbsterhaltung willen zu den Werkzeugen und Waffen zweckrationalen Handelns greift, so ist sie schon nicht mehr Vernunft in der existenzphilosophisch intendierten Bedeutung. Hier zeigt sich meines Erachtens, wohin es führt, wenn Vernunft, von praktischen Zwecken gelöst, auf eine ziellose "Kommunikation" festgeschrieben wird. "Vernunft kennt kein Ausruhen in einem Gewußten", lautet ja, wie zu erinnern ist, die ständig wiederholte Devise der Existenzphilosophie (GO, 128). Der Widerspruch zwischen der gegenständlichen Welt wissenschaftlicher Vernunft, in der es strategisch und zweckrational zugeht, und der unbestimmbaren Welt einer Vernunft, in der alles, auch das Fragen und Denken selbst noch in Frage gestellt werden, ist zu schroff. Er schließt jene idealistische Vermittlung aus, in der Vernunft und Verstand noch aufeinander verwiesen haben. Wo die Vernunft in die Transzendenz abwandert, dort bleibt keine Hoffnung für eine vernünftige Praxis, da diese von vornherein schon unter dem Gesetz der Berechenbarkeit steht.

Wenn die Praxis aber - im Unterschied zur Poiesis - wert- und das heißt norm-orientiert verläuft, so ist sie schon jenen anweisenden Sätzen unterworfen, die Jaspers dem System eines philosophiefernen, weil 
interessegebundenen Tuns zuordnet. Deshalb ist für ihn auch die Weigerung der Wissenschaftler, an der Produktion der Atombombe mitzuarbeiten, noch nicht vernünftig an und für sich. Nach seinem Urteil ist das nur eine Reaktion innerhalb eines in zweckrationalen Grenzen befangenen Planungsdenkens, die zwar von vernünftigen Absichten geleitet, aber selbst noch kein Akt der Vernunft ist.

Die "neue Denkungsart", die der Wissenschaftler - Jaspers beruft sich hier auf Albert Einstein - fordert, darf nicht als pure Alternative zum Wissenschaftsdiskurs aufgefaßt werden. Sie muß vielmehr den Denkenden "umwenden" oder "verwandeln" - wie es in Anlehnung an den Sprachgebrauch mystischer Erfahrungen heißt. In der "Umwendung" kehrt das Denken sich von der Gewohnheit ab, etwas, einen Gegenstand der Erkenntnis, haben zu wollen. Es hat also die Bahn der zweckrationalen Logik verlassen und existiert allein in der Suche nach einem "Wissen" des Ganzen, das dem partikularen Wissen zugrundeliegt. Dieses zu suchende, von Jaspers auch "Grund- oder Fundamentalwissen" genannte "Wissen" ist jedoch nicht mit den Merkmalen des in wissenschaftlicher Sprache codifizierten Wissens ineins zu setzen.

Das Grundwissen ist ohne Gehalt [heißt es dazu in Jaspers' philosophischer Summe], da es nur die Form aller Gehalte bewußt werden läßt. Es ist Gehalt nur durch das Bewußtsein der Weite der Möglichkeiten (GO, 307f.).

Ein so definiertes Fundamentalwissen ist das Gegenteil bestimmten Wissens. Auch hier stoßen wir wieder (wie in der Diskussion des Freiheitsbegriffs) auf die Negativität als den Grund für einen radikal neuen Anfang.

Was heißt aber "Negativität des Wissens"? Auf keinen Fall dessen grundsätzliche Abwertung oder bestimmte Widerlegung. Es geht ja weder um eine neue Irrationalität noch um ein berichtigtes Wissen. Der Begriff des "Grundwissens" könnte zunächst einmal bedeuten, den Grund zu wissen, aus dem diese oder jene Sache so oder so beschaffen ist. So wie der Wissenschaftler fragt: Warum hat dieser bestimmte Körper solche und keine anderen physikalischen Eigenschaften? Eine solche wissenschaftliche Fragestellung via causalitatis schließt jedoch das gegenstandslose "Grundwissen" ausdrücklich aus. Was hier "Grund" heißt, das ist ebensowenig mit dem Verstand zu fassen wie der 
andere Teil des Compositums ("Wissen"). Der Verstand denkt immer zweierlei: den Grund und das Begründete. Von der Beobachtung des Begründeten (Gegenstand) ausgehend, fragt er zum Beispiel: "Woher?" oder "Warum?" Die Indifferenz von Grund und Begründetem zu denken, ist daher nicht Sache des Verstandes sondern allenfalls der Vernunft - wenn hier von "Sache" überhaupt noch die Rede sein kann. Denn das Denken dieser Indifferenz ist ja leer, keine gegenständliche Erfahrung kann ihm entsprechen. In recht umständlicher Weise hieß es dazu schon in der Philosophie:

Das Scheitern des Gedankens in der Gegenstandslosigkeit der Identität von Sein und Grund des Seins ist wiederum die Erscheinung des Seins im Denken eines Nichtdenkbaren (P, 720).

Also stößt das Denken an seiner Grenze auf das Fürwahrhalten, oder den Glauben. Denn das Sein, das seinen Grund in sich selbst hat, das unbedingte oder absolute Sein, ist nur eine schwerfällige philosophische Umschreibung für den Namen jenes personifizierten Ineffabile, das sich schon im Alten Testament mit den Worten vorgestellt hat: "Ich bin, der ich bin."

Natürlich predigt Jaspers nicht den Glauben an einen bestimmten, gar alttestamentarischen Gott. Und doch ist die Konvergenz zwischen Philosophie und Theologie in seiner "Metaphysik" nicht zu übersehen. $\mathrm{Ja}$ das hin und wieder verwendete Bild vom flehentlichen Ausstrecken der Hände nach dem "Grundwissen" ist durchaus als Gebet um die Gnade eines möglichen "Selbstseins" deutbar. Wir berühren hier indessen ein Thema, das den Umkreis unserer Ausgangsfrage überschreitet. Es soll hier genügen, die Grenzen der Wissenschaft bis dahin zu verfolgen, wo sie gleichsam wie Schwellen aus unwegsamem Gelände hervortreten. Ein Standort, von dem aus das möglich sein soll, liegt aber so verstehe ich Jaspers - in einem Glauben, der, wie wir gehört haben, ja nicht nur dem Wissen entgegengesetzt ist, sondern der dieses Wissen als "Fundamentalwissen" umgreift. Umgriffen, umfasst wird aber ein Ganzes, also etwas, das eben nicht - wie die Gegenstände des wissenschaftlichen Wissens - analytisch als etwas Teilbares wahrgenommen und systematisch konstruiert bzw. rekonstruiert werden kann. Insofern verweist das "Grundwissen" auch auf die Abwesenheit jenes Grundes, auf dem die Welt in traditioneller Sicht als ein Ganzes, als 
Kosmos, erfahren worden ist. Auch unter dem Gesichtspunkt der Tradition bezeichnen Begriffe wie "Grundwissen" und "Glaube" einen Mangel in den perspektivisch gebrochenen Erkenntnisbeständen der wissenschaftlichen Diskurse.

Es findet sich in Jaspers' späten zeitkritischen Schriften öfters die Aussage, daß die "Gemeinschaft der Vernünftigen", die imstande sei, die schlimmen Folgen des Wissenschaftsaberglaubens aufzuhalten, ein Gegenstand der Hoffnung bleiben müsse. Diese "Gemeinschaft" entsteht in Jaspers' Konzept nicht durch Verabredung oder gar Organisation, sie ist vielmehr ein Ergebnis des subjektiven Fürwahrhaltens, des Glaubens, der den objektiven Gewißheiten die Idee eines nichtdenkbaren Gegenstandes entgegenhält. Das läßt sich auch paradoxer formulieren: Vom Denken des Nichts geht die Rettung aus. Daß diese Auffassung nur appellativ mitgeteilt werden kann, hängt mit der subjektiven Zulänglichkeit des Fürwahrhaltens zusammen. Deshalb wiederholt Jaspers auch in vielen Wendungen den Satz, die Umkehr könne nur beim einzelnen beginnen. Das ist konsequent, denn die Botschaft von der Rettung aus dem und durch das Nichts ist nicht mehr rational diskutierbar. Man kann sich ihr anschließen oder sie skeptisch verwerfen - das ist einerlei.

Für die Frage nach einer konkreten Begrenzung der Bedrohungen, die vom politisch sanktionierten Mißbrauch wissenschaftlichen und technischen Wissens ausgehen, ist diese Botschaft letztenendes belanglos. Oder sollen wir uns vertrauensvoll dem Glauben hingeben, $\mathrm{da} \mathrm{B}$ mit dem Nachdenken über die Grenzen der Wissenschaften hier und jetzt schon die Gemeinschaft der Vernünftigen begonnen hätte? Wir können das nicht wissen, wir können das nur im Vollzug erfahren. Darin liegt zweifelsfrei eine Aporie, die für die Existenzphilosophie konstitutiv ist. Nämlich die Verlegenheit, gemeinsam über unsere Situation philosophieren zu wollen, ohne jemals die Gewißheit zu haben, ob es überhaupt eine Möglichkeit gibt, einen riskanten Handlungsplan zu reparieren oder ganz über Bord zu werfen. 
Literatur

\section{Karl Jaspers}

Psychologie der Weltanschauungen, München/Zürich [1919]1985

Die Idee der Universität, Berlin 1923 und 1946

Die geistige Situation der Zeit, Berlin [8.Aufl.] 1979

Vom Ursprung und Ziel der Geschichte, München/Zürich [8.Aufl.] 1983

AZM Die Atombombe und die Zukunft des Menschen, München/Zürich [7.Aufl.] 1983

GO Der philosophische Glaube angesichts der Offenbarung, München/Zürich [3.Aufl.] 1984

P Philosophie, Berlin/Göttingen/Heidelberg [2.Aufl.] 1948

PW Psychologie der Weltanschauungen, Berlin [9.Aufl.] 1973

Arendt, Hannah u. Jaspers, Karl: Briefwechsel 1926-1969, hrsg. von L.Köhler/H.Saner, München/Zürich 1985

Beck, Ulrich: Risikogesellschaft. Auf dem Weg in eine andere Moderne, Frankfurt a.M.1986

Fahrenbach, Helmut: Das "philosophische Grundwissen" kommunikativer Vernunft - Ein Beitrag zur gegenwärtigen Bedeutung der Philosophie von Karl Jaspers. In: Karl Jaspers. Philosoph, Arzt, politischer Denker, hrsg. von J.Hersch et al., München/Zürich 1986, S.232-280

Harth, Dietrich: Die Krise der modernen Wissenschaften im Denken Karl Jaspers'. In: Jaspers, témoin de son temps, hrsg.v. G.Merlio, Bordeaux 1986, S.77-92

Harth, Dietrich: Die Kritik der existenzphilosophischen Kulturkritik: Karl Jaspers' "Die geistige Situation der Zeit" im Kontext der frühen 30er Jahre. In: Situation de l'homme et histoire de la philosophie dans l'oeuvre de Karl Jaspers, hrsg.v.J.-M.Paul, Nancy 1986, S.137-152

Henrich, Dieter: Denken im Blick auf Max Weber. In: Karl Jaspers. Philosoph, Arzt, politischer Denker, hrsg.v.J.Hersch et al., München/Zürich 1986, S.207-231

Weizsäcker, Carl Friedrich von: Die Verantwortung der Wissenschaft im Atomzeitalter, Göttingen 1957 